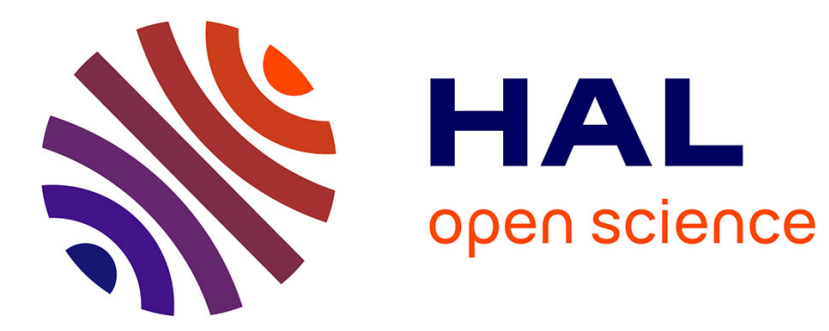

\title{
Petites communes et opérateurs privés : généalogie du 'modèle français' de gestion des services d'eau potable
} C. Pezon, Guillem Canneva

\section{To cite this version:}

C. Pezon, Guillem Canneva. Petites communes et opérateurs privés: généalogie du 'modèle français' de gestion des services d'eau potable. Espaces et sociétés (Paris, France), 2009, 10.3917/esp.139.0021. halshs-02549395

\author{
HAL Id: halshs-02549395 \\ https://shs.hal.science/halshs-02549395
}

Submitted on 21 Apr 2020

HAL is a multi-disciplinary open access archive for the deposit and dissemination of scientific research documents, whether they are published or not. The documents may come from teaching and research institutions in France or abroad, or from public or private research centers.
L'archive ouverte pluridisciplinaire HAL, est destinée au dépôt et à la diffusion de documents scientifiques de niveau recherche, publiés ou non, émanant des établissements d'enseignement et de recherche français ou étrangers, des laboratoires publics ou privés. 


\title{
Petites communes et opérateurs privés: généalogie du 'modèle français' de gestion des services d'eau potable
}

\author{
Dr Christelle Pezon (IRC), Guillem Canneva (APT)
}

\section{Introduction}

En France, la desserte en eau potable du territoire est quasi-généralisée depuis la fin des années 1980 : moins de $1 \%$ de la population n'est pas raccordé au réseau public (IFEN, 2007). Pour 75\% de la population, le service public d'alimentation en eau potable est délégué à un opérateur privé. Ce modèle de gestion «à la française », qui a été exporté avec plus ou moins de réussite, concentre l'attention dans un contexte marqué par l'opposition entre gestion publique et privée. Il occulte de fait la grande diversité des services d'eau, tant organisationnelle que managériale, voire leur grande complexité, née de la combinaison possible d'organisations et de modes de gestion distincts pour chacune des activités (production, distribution), de l'application de règles différentes selon la taille des services et de l'évolution territoriale que ces derniers connaissent depuis la loi Chevènement (1999).

Comment comprendre la domination du modèle en délégation de service public (DSP) ? Elle apparaît comme l'aboutissement d'une histoire, où l'organisation institutionnelle et territoriale et les opportunités de gestion ont joué un rôle déterminant. La mise en perspective historique des contraintes auxquelles les collectivités locales ont été soumises, ainsi que des options qu'elles ont retenues pour organiser et gérer leurs services d'eau potable, permet d'analyser l'ensemble des ressorts du modèle actuel et d'envisager ses possibles évolutions.

Nous nous intéresserons d'abord à l'échelle territoriale d'organisation des services d'eau (1) puis aux modalités de leur gestion (2). Nous analyserons enfin l'évolution conjointe de leur organisation et de leur gestion (3). 


\section{Organisation des services d'eau potable: fragmentation communale et tentatives de rationalisation}

Les services d'eau potable en France peuvent être administrés à l'échelle communale ou être confiés par les communes à des établissements publics de coopération intercommunale (EPCI), appellation qui regroupe des formes juridiques variées : syndicats intercommunaux, syndicats mixtes ou encore communautés. En outre, les activités de production et de distribution peuvent être confiées à des collectivités différentes.

Leur grand nombre (12 000 services) et leur grande diversité s'enracine dans l'extrême fragmentation des communes et dans la possibilité de dissocier les activités concourant à l'alimentation en eau potable (1.1). La rationalisation du territoire administratif local recherchée par la loi Chevènement se concrétise par la réduction du nombre de services d'eau potable (1.2).

\subsection{L'héritage du découpage communal dans l'organisation des services d'eau}

Cette pléthore de services est un héritage du grand nombre de communes que compte la République. Politiques et bureaucrates ont régulièrement dénoncé l'inadaptation et l'archaïsme de la carte communale au regard des problèmes liés au développement urbain ou économique de la France, mais les tentatives pour réduire par la fusion le nombre des communes se sont immanquablement soldées par des échecs si bien que la voie de la coopération intercommunale est finalement apparue comme la solution la plus prometteuse pour les tenants de la rationalisation administrative ${ }^{1}$.

Après l'échec de l'introduction autoritaire des municipalités de canton par la Constitution de l'an III, les bases d'une coopération intercommunale furent timidement posées par la loi du 18 juillet 1837 . Suite à la loi du 5 avril 1884, qui fonde les municipalités élues contemporaines, la loi du 22 mars 1890 introduit le syndicat intercommunal qui constitue, jusqu'en 1959, l'unique organisation à laquelle les communes peuvent transférer une compétence et une seule (syndicat intercommunal à vocation unique, SIVU). Les ordonnances du 5 janvier 1959 apportent des transformations importantes pour les syndicats intercommunaux $\left(\mathrm{n}^{\circ} 59-29\right)$ et instituent la formule du district urbain $\left(\mathrm{n}^{\circ} 59-30\right)$. Les syndicats pourront désormais cumuler des compétences (syndicat intercommunal à vocation multiple, SIVOM) et associer communes, syndicats de communes et départements (syndicat mixte, SM). Les villes disposeront avec le district urbain d'une organisation adaptée à la gestion complexe de leur développement. A partir de 1966, elles peuvent aussi s'organiser en communautés urbaines, auxquelles les compétences communales en eau potable et en assainissement sont obligatoirement transférées. La loi Chevènement de 1999 clôt provisoirement la liste déjà longue des organisations susceptibles d'exercer une responsabilité sur les services d'eau. Elle crée deux nouvelles communautés, l'une destinée aux communes rurales (communauté de communes, CC), l'autre aux agglomérations moyennes (communauté d'agglomération, $\mathrm{CA}$ ), et réserve la communauté urbaine aux centres urbains les plus peuplés. ${ }^{2}$

Très tôt, les communes ont satisfait leur mission d'hygiène, à l'origine de leur responsabilité en eau potable (Duroy, 1996), en s'organisant à plusieurs. Le premier syndicat intercommunal d'eau potable voit le jour en Ardèche, en 1907 : il regroupe quatre communes qui mettent en commun leurs travaux d'adduction et l'entretien d'un réservoir. Le premier service intercommunal de production d'eau potable est né. En 1936, 290 syndicats d'eau potable, responsables de la production et/ou de la distribution aux abonnés, regroupent 1641 communes (Leydet, 1936). Après la nationalisation du secteur énergétique en 1946 et la disparition d'un grand nombre de syndicats d'électrification, la compétence en eau devient le principal moteur de l'intercommunalité.

En 1988, il existe plus de 15400 EPCI, dont près de 14500 sont des syndicats intercommunaux : un quart est compétent en eau potable, dont une très large majorité $(88 \%)$ à l'exclusion de toute autre compétence (GIP

\footnotetext{
${ }^{1}$ Voir notamment Bourjol (1993) et Perrin (1994) pour la période antérieure à la loi Chevènement

${ }^{2}$ La communauté de communes regroupe des communes sans critère de taille de population. La communauté d'agglomération regroupe des communes dont une de plus de 15000 habitants sur une zone de plus de 50000 habitants. La communauté urbaine regroupe des communes dont une de plus de 50000 habitants sur une zone de plus de 500000 habitants (il en existe de plus petites, crées avant la loi de 1999).
}

Espaces et Sociétés - Usages et Régulations des Eaux Urbaines 139 n4/2009 pp.21-38 
Reclus, 1992). C'est donc essentiellement dans le cadre de SIVU, forme exclusive de regroupement jusqu'en 1959, qu'une majorité de communes a organisé ses services, des plus petites aux plus grandes, les organisations à vocation urbaine (district et communauté urbaine) étant peu prisées des collectivités locales.

Tableau 1. Les services communaux et intercommunaux d'eau potable en 1988

\begin{tabular}{|l|l|l|l|l|l|l|}
\hline Types d'organisation & Communes & SIVU & SIVOM & SM & District & CU \\
\hline Nb d'organisation* & 37200 & 12200 & 2280 & 750 & 165 & 9 \\
\hline Nb de services d'eau potable** & 11800 & 3375 & 455 & nd & 62 & 9 \\
\hline
\end{tabular}

A la veille de la loi Chevènement, la carte administrative locale est brouillée par le chevauchement de 37200 communes et 15400 EPCI, le plus souvent des SIVU, auxquels les communes adhèrent de préférence à des EPCI plus intégrés qui les videraient d'une part substantielle de leur pouvoir. Pour organiser leurs services d'eau potable, les communes peuvent découpler les activités de production et de distribution : elles transfèrent alors la maîtrise d'ouvrage pour la production d'eau potable à un syndicat et conservent une maîtrise d'ouvrage communale pour la distribution d'eau. En 2001, l'IFEN estime que 30\% des communes organisent encore différemment les deux activités (l'une communale et l'autre syndicale ou les deux activités en syndicat mais par des syndicats différents).

\subsection{La 'révolution silencieuse' 3 des communautés et l'organisation des services d'eau potable}

Les EPCI à fiscalité propre proposés par la loi Chevènement se constituent sur la base d'un projet de développement qui intègre, à l'échelle du territoire, des compétences très larges, certaines obligatoires, d'autres optionnelles ou facultatives. Ces communautés ont été plébiscitées par les communes : 2588 communautés rassemblent, au $1^{\mathrm{er}}$ janvier 2007, 33413 communes représentant 54,2 millions d'habitants. Alors que les précédentes tentatives ${ }^{4}$ avaient échoué dans l'accompagnement des mutations urbaines du 20 ème siècle, les communautés à vocation urbaine de la loi Chevènement ont rencontré un succès qui doit s'analyser dans le contexte européen de mise en concurrence des territoires. En zone rurale, la rationalisation est aussi d'importance : les communautés de communes (CC) réunissent en moyenne 12,5 communes et plus de 11000 habitants.

Les 14 communautés urbaines sont obligatoirement compétentes en eau potable. Elles représentent plus de 6 millions d'habitants. La moitié des 169 communautés d'agglomération et moins de $9 \%$ des 2400 communautés de communes ont retenu la compétence en eau.

Les deux tiers des services d'eau potable sont organisés à l'échelle communale. Ils concernent $28 \%$ de la population (Tableau 2). Un tiers des services est organisé en EPCI. Pour les trois-quarts d'entre eux, l'eau est une compétence exclusive : plus de $55 \%$ des communes (représentant près d'un Français sur trois) organisent leur service d'eau potable dans le cadre d'un SIVU.

\footnotetext{
${ }^{3}$ Cette expression est empruntée à M. DALLIER, sénateur de la Seine-Saint-Denis (Rapport d'information au Sénat, $\mathrm{n}^{\circ}$ 193, 2005-2006)

${ }^{4}$ Celle de 1959 avec la création du district urbain (mention finalement abandonnée en 1970 compte tenu du caractère rural des communes qui l'utilisaient), celle de 1966 avec la communauté urbaine qui, après 30 ans, a abouti à la création de 5 CU volontaires sur la centaine escomptée, et enfin, celle de 1992 qui crée la communauté de villes (on en compte seulement 5 en 1999).
}

Espaces et Sociétés - Usages et Régulations des Eaux Urbaines 139 n4/2009 pp.21-38 
Tableau 2. Organisation des services d'eau potable en 2007

\begin{tabular}{|l|l|l|l|l|l|l|l|l|}
\hline Organisation & Commune & CC & CA et SAN* & CU & SIVU & SIVOM & SM & Total \\
\hline Nb de services & \multirow{2}{*}{8074} & 207 & 85 & 14 & 2949 & 374 & 143 & 11846 \\
\cline { 2 - 9 } Nb de communes & 2281 & 1242 & 358 & 19762 & 3003 & 2060 & 367805 \\
\hline Population (part) & $28 \%$ & $4 \%$ & $16 \%$ & $10 \%$ & $28 \%$ & $6 \%$ & $8 \%$ & $100 \%$ \\
\hline
\end{tabular}

Source : à partir de Canneva, 2007. *Syndicat d'agglomération nouvelle.

Si les formes traditionnelles d'organisation des services (commune, SIVU) conservent leur leadership ( $93 \%$ des services, $75 \%$ des communes et $56 \%$ de la population), elles déclinent simultanément : près de 3800 services communaux (34\%) et plus de 400 SIVU (13\%) ont disparu en 20 ans. De leur coté, les communautés desservent en 2007 une population égale à celle qui est alimentée par des services communaux ou par SIVU (environ 17 millions d'habitants). Environ $10 \%$ des communes ont organisé leurs services d'eau dans le cadre communautaire : leurs 306 services d'eau assurent la distribution d'eau potable à près d'un Français sur trois.

L'émergence récente des communautés introduit deux ruptures majeures. La première est historique : pour la première fois, le nombre de services d'eau potable se réduit. Estimé à environ 15000 en 1988, il est stable jusqu'en 2001 (d'après l'IFEN) et se réduit à moins de 12000 en avril 2007 (d'après le ministère de l'Intérieur) soit une réduction de $20 \%$ en 6 ans. La seconde est organisationnelle : les modalités d'exercice de la compétence en eau potable sont très différentes selon le type d'organisation. Communale ou syndicale, la compétence peut se limiter à la production ou à la distribution d'eau potable. Communautaire, la compétence est intégrée et la maîtrise d'ouvrage unique. Les décisions relatives à la conservation et à l'amélioration du patrimoine technique (du captage au compteur) relèvent alors d'une seule collectivité, la communauté, à laquelle est transférée la totalité des actifs, des dettes et des personnels attachés aux services de production et/ou de distribution précédemment en activité à l'échelle de son territoire.

\section{Modes de gestion des services d'eau potable}

Nous venons de voir qu'un service d'eau peut s'organiser au niveau de la commune et/ou d'une intercommunalité, et qu'une très large majorité de communes a opté pour une organisation intercommunale. Nous allons voir à présent que les services d'eau sont assujettis à des règles de gestion différenciées selon leur taille (2.1.) et qu'une très large proportion de la population est desservie par des services délégués à un opérateur privé (2.2.).

\subsection{Etre ou ne pas être assujetti à la règle de l'équilibre budgétaire}

Le nombre de collectivités qui a la faculté de financer via l'impôt leurs services d'eau s'est progressivement réduit. Les premières restrictions datent de la fin des années 1960 : les instructions comptables de 1967 et 1969 commandent aux collectivités de plus de 10000 habitants d'isoler les recettes et les dépenses de leurs services d'eau dans un budget annexé à leur budget général, et d'équilibrer leurs dépenses d'exploitation et d'investissement en facturant le service rendu. En 1982, les lois de décentralisation interdisent à toutes les communes de financer via l'impôt leurs services publics industriels et commerciaux. Cette mesure ne peut s'appliquer aux services d'eau potable qu'après l'ordonnance du 2 décembre 1986 -relative à la liberté des prix et de la concurrence- qui abolit l'ordonnance du 30 juin 1945 sur laquelle s'appuyait l'Etat pour réglementer le prix de l'eau'. La déréglementation du prix de l'eau sera de courte durée : dès janvier 1988, une loi d'amélioration de la décentralisation prévoit des exceptions au régime général défini en 1982. Ainsi (loi n88-13 du 5/1/1988 art.14) 'lorsque les exigences du service public conduisent la collectivité à imposer des contraintes particulières de fonctionnement' ou quand 'le fonctionnement du service public exige la réalisation

\footnotetext{
${ }^{5}$ Le surnombre est dû à l'appartenance de certaines communes à plusieurs EPCI.

${ }^{6}$ Le prix de l'eau était d'ailleurs bloqué depuis la fin des années 1970
} 
d'investissements qui, en raison de leur importance et eu égard au nombre d'usagers, ne peuvent être financés sans augmentation excessive des tarifs' ou encore 'lorsque, après la période de réglementation des prix, la suppression de toute prise en charge par le budget de la commune aurait pour conséquence une hausse excessive des tarifs', les communes pourront abonder le budget de leurs services d'eau. Avec la dernière instruction comptable applicable aux services d'eau et d'assainissement de 1995 (appelée M49), les communes de moins de 3000 habitants et les EPCI dont les communes membres comptent chacune moins de 3000 habitants sont durablement affranchis de la contrainte de l'équilibre budgétaire de leur service. La contribution des budgets généraux au financement des investissements est estimée globalement à 56 millions d'euros par an (BIPE/FP2E, 2008).

Le Tableau 3 indique que $89 \%$ des services d'eau communaux comptent moins de 3000 habitants. Ces services représentent 3,9 millions d'habitants, soit 19\% de la population alimentée par un service communal, et sont dispensés d'équilibrer leurs dépenses avec les redevances perçues auprès des abonnés, quel que soit leur mode de gestion.

Tableau 3. Services assujettis ou non à la règle de l'équilibre budgétaire (hors CA et CU) ${ }^{7}$

\begin{tabular}{|c|c|c|c|c|c|c|c|}
\hline & & $\begin{array}{c}\text { Service } \\
\text { communal }\end{array}$ & $\mathrm{CC}$ & SIVU & SIVOM & $\begin{array}{c}\text { Sous-total } \\
\text { EPCI }\end{array}$ & Total \\
\hline \multirow{3}{*}{$\begin{array}{l}\text { Non } \\
\text { assujetti }\end{array}$} & $\mathrm{Nb}$ de services & \multirow{2}{*}{7015} & 107 & 2378 & 257 & 2742 & 9757 \\
\hline & $\begin{array}{l}\mathrm{Nb} \text { de } \\
\text { communes }\end{array}$ & & 1076 & 14636 & 2010 & 17722 & 24737 \\
\hline & $\begin{array}{l}\text { Population } \\
\text { (millions) }\end{array}$ & 3,9 & 0,5 & 6,9 & 1 & 8,4 & 12,3 \\
\hline \multirow{3}{*}{ Assujetti } & $\mathrm{Nb}$ de services & \multirow{2}{*}{855} & 100 & 570 & 117 & 787 & 1642 \\
\hline & $\begin{array}{l}\mathrm{Nb} \text { de } \\
\text { communes }\end{array}$ & & 1241 & 6584 & 1645 & 9470 & 9470 \\
\hline & $\begin{array}{l}\text { Population } \\
\text { (millions) }\end{array}$ & 16 & 2,3 & 13,4 & 3,3 & 19 & 35 \\
\hline
\end{tabular}

Source : d'après Canneva, 2007 ; données Ministère de l'intérieur, avril 2007

Le Tableau 3 indique également que 2742 EPCI dont 2378 SIVU ne sont pas assujettis à la règle de l'équilibre budgétaire, quel que soit leur mode de gestion. Ils concernent 17722 communes et près de 8,5 millions d'habitants. Si l'on considère globalement les services communaux et les services intercommunaux, 9757 services, soit plus de $80 \%$, ne sont pas assujettis à la règle de l'équilibre budgétaire. Ils concernent 24737 communes et plus de 12,3 millions d'habitants, soit un Français sur cinq. Autrement dit, moins de $20 \%$ des services d'eau doivent équilibrer leurs dépenses grâce aux factures acquittées par leurs abonnés, quel que soit leur mode de gestion.

Une lecture plus fine du type d'EPCI concerné montre que près de $81 \%$ des SIVU sont exemptés de l'équilibre budgétaire de leurs services d'eau alors que la moitié des communautés de communes et la totalité des autres communautés doivent au contraire équilibrer leurs dépenses sur la base des redevances perçues auprès des abonnés. Aussi la réorganisation des services d'eau potable en communautés n'est-elle pas neutre en termes de gestion : des services précédemment exemptés d'une tarification au coût complet de l'eau potable basculent dans

\footnotetext{
${ }^{7}$ Par construction, les services d'eau gérés par les communautés d'agglomération et les communautés urbaines sont soumis à la règle de l'équilibre budgétaire.
}

Espaces et Sociétés - Usages et Régulations des Eaux Urbaines 139 n4/2009 pp.21-38 
la catégorie des 'grands' services pour lesquels un financement partiellement fiscal des investissements doit demeurer exceptionnel.

\subsection{L'affermage des services d'eau potable : compromis public - privé}

Les collectivités locales peuvent, soit gérer elles-mêmes leurs services (régie), soit les déléguer à un opérateur privé (délégation de service public). Les mêmes options de gestion s'offrent à l'EPCI auquel elles transfèrent toute ou partie de leur compétence en eau potable.

La délégation de service public (DSP) domine : elle concerne 75\% de la population et des volumes d'eau distribuée, et plus d'une commune sur deux. Elle est toutefois minoritaire en nombre de services puisque la FP2E ${ }^{8}$ revendique, en 2007, la gestion déléguée de 4814 services (BIPE/FP2E, 2008) contre plus de 7000 régies, pour la plupart communales et rurales, mais aussi communautaires et urbaines (CU de Strasbourg par exemple) qui desservent 15 millions d'habitants en eau potable.

Pour comprendre la gestion des services d'eau potable, il convient de ne pas assimiler gestion déléguée et privatisation mais plutôt de considérer la DSP comme un compromis entre gestion publique et gestion privée. En effet, la DSP procède très largement par contrat d'affermage et repose donc sur un financement public des services d'eau. L'opérateur fermier est chargé d'exploiter le service pour le compte de la commune ou de l'EPCI, pour une durée moyenne qui s'est réduite à 11 ans. Il perçoit auprès des abonnés l'intégralité des factures d'eau et conserve la part supposée couvrir ses coûts d'exploitation et sa marge, tels que définis contractuellement. Il reverse à l'autorité délégante la part supposée couvrir les coûts qu'elle finance, essentiellement les coûts d'investissement dans les infrastructures, les opérateurs en assumant seulement $13 \%{ }^{9}$.

Tableau 4. Financement des infrastructures d'eau potable et d'assainissement

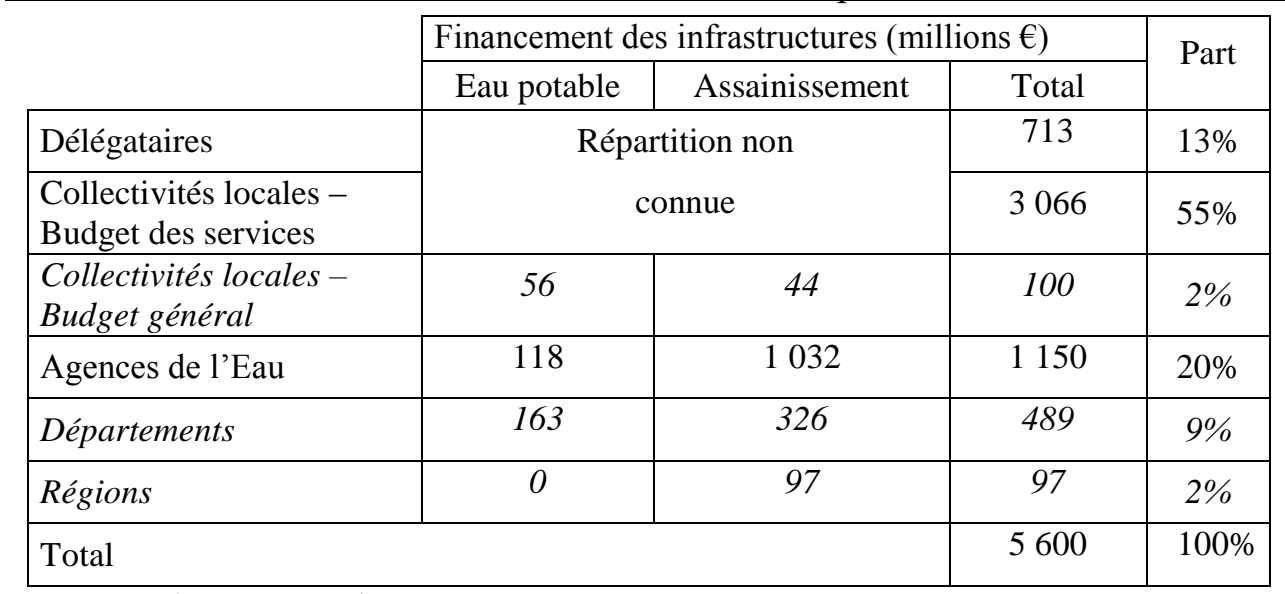

Source : d'après BIPE/FP2E, 2008

Les premiers financeurs sont les collectivités locales (près de 50\%) puis, pour l'eau potable, les départements et, pour l'assainissement, les agences de l'eau. Les financements trouvant leur origine dans les ressources fiscales (en italique) représentent une part minoritaire (13\%), face à ceux liés à la facture d'eau (part collectivité et redevance des agences), ce qui montre l'application quasi-intégrale du principe de recouvrement complet des coûts, cher à l'Union Européenne.

La gestion en affermage se distingue radicalement d'une privatisation parce qu'elle s'accorde avec une propriété publique des infrastructures, s'appuie sur un financement public des investissements et s'avère même compatible avec un financement fiscal de ces derniers, lorsque les services ne sont pas assujettis à l'équilibre budgétaire.

\footnotetext{
${ }^{8}$ Fédération professionnelle des entreprises de l'eau

${ }^{9}$ Cette part correspond en général aux investissements amortissables dans la durée des contrats, c'est-à-dire ceux qui s'usent vite.
}

Espaces et Sociétés - Usages et Régulations des Eaux Urbaines 139 n4/2009 pp.21-38 


\section{L'affermage des services d'eau potable : compromis historique entre solidarité et efficacité}

La domination du contrat d'affermage est le fruit d'un processus historique, intimement lié à l'organisation territoriale des services et aux règles de gestion, qui a débuté par la concession, pendant la seconde moitié du $19^{\text {ème }}$ siècle, remplacée par la régie simple, des années 1910 aux années 1970, avant que l'affermage ne prenne le pas en termes de population (1973) puis de communes (1980).

\subsection{Grandeur et décadence de la concession}

Jusqu'à la Grande Guerre, la concession et la régie simple sont les seules modalités dont disposent les communes et les syndicats pour gérer leurs services d'eau potable. ${ }^{10}$ Elles s'opposent en tout point. Le concessionnaire s'engage à développer un service d'eau en contrepartie de l'exploitation exclusive de la distribution d'eau sur le territoire communal, pour une durée calée sur l'espérance de vie des équipements (60 à 99 ans). Le concessionnaire se rémunère à travers la facturation du service aux abonnés. Les tarifs s'établissent selon le principe d'un recouvrement complet des coûts (définis ex ante), marge de l'opérateur incluse. A l'opposé, la régie simple, est un service communal dont les investissements sont financés sur le budget général de la collectivité, donc par l'impôt, et ne sont astreints à aucune contrainte de rentabilité. Les abonnés peuvent être mis à contribution, notamment pour les travaux de branchement, mais les recettes d'exploitation contribuent à titre secondaire aux besoins de financement des services. Le choix de la concession ou de la régie révèle la conception qu'ont les communes et les syndicats du service d'eau potable à domicile, entre service marchand élitiste ou nécessité universelle.

Au milieu du $19^{\text {ème }}$ siècle, l'eau à domicile est encore un privilège d'Ancien Régime (Goubert, 1987). Pour assurer leur mission de salubrité, les communes cherchent d'abord à garantir la mise à disposition d'eau aux établissements publics (administratifs, hôpitaux, écoles) et aux bornes-fontaines qui forment alors le 'service public des eaux'. Avec le 'Traité de concession', les communes trouvent un moyen de financer le développement du service public, en offrant au concessionnaire le monopole de la desserte en eau à domicile (le 'service privé' de distribution d'eau). Les communes concédantes acquittent chaque année un 'abonnement' aussi appelé 'garantie d'intérêt' qui permet un amortissement sur 20 à 25 ans des travaux estimés ex ante par le concessionnaire pour développer la distribution d'eau aux bornes-fontaines et aux établissements publics ; elles organisent ainsi une distribution d'eau salubre sans s'endetter et à prix coûtant. Le bénéfice espéré de l'entreprise provient de la vente exclusive d'abonnements au service privé. Alors que l'eau des bornes fontaines est gratuite, l'accès à domicile est assumé par chaque abonné. La solidarité s'exerce alors des citadins aisés vers les plus modestes : ce sont les abonnements au service privé qui doivent rémunérer le risque industriel et financier pris par le concessionnaire (Pezon, 2000).

A partir des années 1880 , la conjonction de trois facteurs s'avère propice à une révision par les communes des ambitions qu'elles s'étaient initialement fixées pour satisfaire leur mission de salubrité. Dans la lignée des découvertes de Pasteur, les hygiénistes alertent sur l'origine hydrique de maladies mortelles et font pression pour que l'usage de l'eau se répande parmi les populations (Murard \& Zylberman, 1996). Un deuxième facteur tient aux progrès scientifiques et techniques réalisés pour, d'une part, dimensionner les réseaux et industrialiser la fabrication de canalisations résistantes à la pression, et, d'autre part, analyser les propriétés biologiques et physico-chimiques de l'eau, pomper et traiter des eaux brutes à grande échelle. Un dernier facteur résulte de la grande loi municipale de 1884 qui instaure un régime de démocratie directe pour l'élection du maire. Les hommes politiques comprennent l'intérêt à rendre plus accessible l'eau à domicile : les gains attendus sont sanitaires, symboliques (l'entrée en modernité à travers l'appropriation d'équipements sophistiqués) et politiques, une très large majorité de la population n'ayant pas accès à l'eau à domicile. En effet, après un demi-

\footnotetext{
${ }^{10}$ Notons que la distribution d'eau fait exception au regard des autres services locaux nés de la Révolution industrielle (gaz, électricité, transport) : ni le Conseil d'Etat, ni l'administration ne se sont jamais opposés à la création, par les communes, de services municipaux des eaux. Voir Duroy, 1996.
}

Espaces et Sociétés - Usages et Régulations des Eaux Urbaines 139 n4/2009 pp.21-38 
siècle de leadership, la concession a permis de desservir en eau à domicile moins de 130000 personnes, dans des villes qui totalisent 4,5 millions d'habitants (Goubert, 1987).

Les villes engagées dans des contrats de concession tentent d'obtenir de leurs concessionnaires qu'ils facilitent l'accès au service privé et qu'ils investissent dans le traitement des eaux préalablement à leur distribution. Les concessionnaires refusent de négocier à la baisse la rémunération de leur activité et portent les litiges devant les tribunaux, confiants dans l'interprétation littérale qui sera faite de leurs contrats. Si la Haute Cour Administrative donne raison aux concessionnaires et les conforte dans les droits obtenus au moment de la signature des Traités, elle disqualifie, auprès des communes, la concession comme arrangement institutionnel susceptible de développer la desserte en eau potable à domicile. Piégées par des contrats de très longue durée, les villes concernées vont, pour les plus grandes d'entre elles, rompre leur concession ${ }^{11}$ et gérer le service en régie simple et, pour les plus petites, accepter de prendre à leur charge les investissements en traitement et en extension de réseaux, transformant de fait progressivement leur contrat de concession en contrat d'affermage (Pezon, 2007).

Les communes qui n'ont pas encore commencé à s'équiper de réseaux de distribution d'eau potable vont s'orienter vers la création de régies des eaux. En 1908, plus de la moitié des villes gèrent leurs services d'eau sous cette forme (Burel, 1912). Après 1918, 'l'exploitation directe des services municipaux par les communes tend à se généraliser', particulièrement pour les services de distribution d'eau que deux villes de plus de 5000 habitants sur trois exploitent en régie (Monsarrat, 1920). La gestion en régie simple est devenue la norme. En 1939, 75\% de la population alimentée en eau potable à domicile est desservie par des services en régie (Loosdregt, 1990). En 40 ans, le taux de desserte en eau potable est passé de moins de 1\% à plus de 63\% (Loriferne, 1987). Les investissements ont été financés grâce aux impôts locaux et à l'inflation - opérant une redistribution des gros contribuables et des détenteurs de capitaux (rentiers) vers les autres.

\subsection{Le temps de l'affermage : échec des communes ou succès de l'Etat ?}

Au début des années 1950, la situation n'a guère changé. En 1954, il existe 9789 services en régie et 817 services en DSP. 31\% des volumes d'eau distribuée en ville sont gratuits, proportion qui atteint $58 \%$ dans les communes rurales concernées. Près de 29000 communes n'ont pas encore organisé de service d'eau potable, représentant 14 millions de personnes (Min. Agriculture, 1959). Puis, en moins de 20 ans, le nombre de personnes desservies par un service en affermage double, égalant, en 1973, la population desservie par une régie. La gestion en affermage augmente brutalement après les lois de décentralisation et jusqu'à la loi Sapin en 1993 (Figure 1).

${ }^{11}$ C'est le cas des villes de Nantes, Lyon, Rouen et Toulon. 
Figure 1. Population desservie par un service délégué ou par une régie (1938 - 1993)

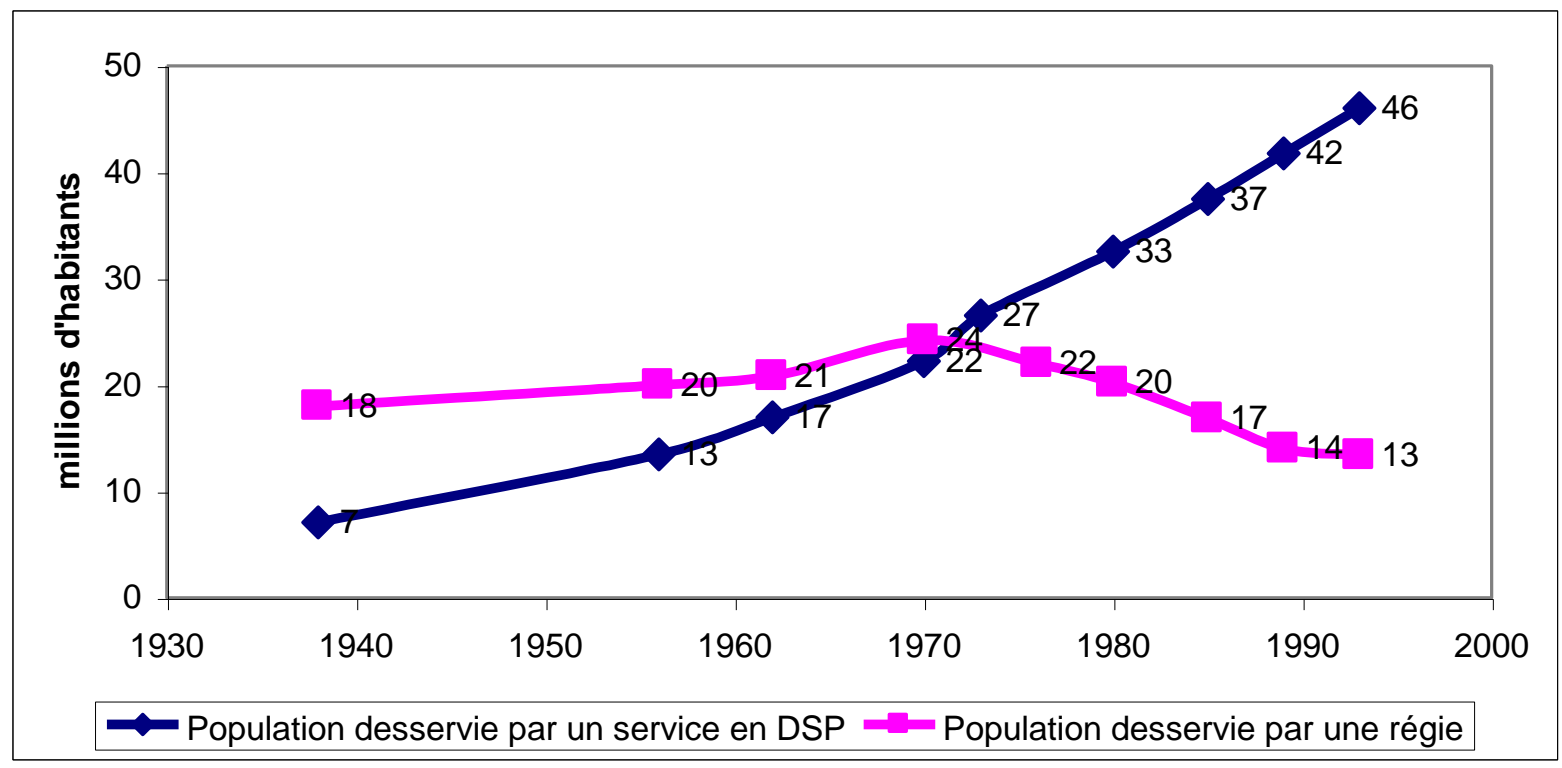

Source : d'après Pezon, 2000

Deux types de communes rurales peinent à organiser leurs services d'eau : celles qui disposent d'eau de bonne qualité et facilement accessible mais qui ne peuvent assumer le coût d'un réseau (Massif Central), et celles qui doivent non seulement développer des réseaux locaux de distribution onéreux mais aussi s'équiper pour produire de l'eau potable (Bretagne et départements situés sur la façade Atlantique). Pour les premières, la difficulté est d'ordre financier ; pour les secondes, la difficulté est financière mais aussi organisationnelle : il faut en effet mutualiser les équipements de production à une échelle intercommunale et disposer des compétences nécessaires à leur exploitation.

La création du FNDAE (Fonds National pour le Développement des Adductions d'Eau) en 1954 doit résoudre la question du financement : la population desservie à domicile (urbaine pour l'essentiel) s'acquittera d'une taxe proportionnelle à sa consommation qui financera l'équipement des communes rurales en alimentation en eau potable. Cette solidarité des urbains vers les ruraux est orchestrée par le ministère de l'Agriculture et ses services déconcentrés. Les ingénieurs d'Etat privilégient l'organisation qui optimise les investissements en fonction des ressources en eau disponibles. Pour bénéficier du FNDAE, les communes rurales doivent se plier à leurs prescriptions en matière de travaux, d'organisation (communale ou syndicale) et de mode de gestion (Pezon, Petitet, 2004).

La taille, en général modeste, de nombreux syndicats et leur compétence limitée réglementairement à l'alimentation en eau potable ne leur permet souvent pas d'atteindre une échelle efficace pour l'exploitation. Pour les communes du Grand Ouest, la gestion en affermage est vivement encouragée : elle s'épanouit dans le cadre du cahier des charges type de 1951 et s'accompagne, dès 1952, d'une régulation administrative des tarifs qui garantit aux élus une évolution du prix de l'eau limitée à celle de l'inflation (Pezon, 2002). Ainsi, pour nombre de communes rurales, l'affermage permet de contourner la difficulté de l'exploitation de services syndicaux, confrontés à des ressources plus difficiles à exploiter et trop petits pour être gérés à leur modeste échelle ${ }^{12}$.

\footnotetext{
${ }^{12}$ Les formes de gestion déléguée en 'régie intéressée' ou en 'gérance', où l'opérateur est rémunéré par la collectivité et non par les usagers du service, sont restées marginales.
} 
A compter de 1969, la taille moyenne des communes qui afferment la gestion de leurs services d'eau augmente de façon significative. ${ }^{13}$ La plus forte variation annuelle se produit en 1973, année où 1460 communes (totalisant 1,77 million d'habitants) passent en affermage. La même année, la population alimentée par des services en régie passe sous la barre des $50 \%$. La gestion en affermage ne progresse plus seulement grâce aux services nouvellement créés mais également aux dépens de la régie simple. Les instructions comptables de 1967 et 1969 imposent l'autonomie budgétaire aux régies urbaines. Seule l'intercommunalité permet les économies d'échelle inhérentes aux services en réseaux, et le report sur les abonnés d'une part significative du coût des services oblige les communes à privilégier l'efficacité. L'affermage permet en outre aux communes de récupérer la TVA payée sur les investissements ${ }^{14}$. Les services qui adhèrent aux EPCI existants adoptent leur mode de gestion, l'affermage de façon prépondérante. Dans les cas où l'intercommunalité n'est pas l'option retenue notamment pour des raisons politiques - les opérateurs privés, implantés sur tout le territoire, offrent la possibilité d'une gestion mutualisée entre plusieurs collectivités.

La dernière poussée de la gestion en affermage se déroule du milieu des années 1980 à 1993. En 1980, 79\% des villes de plus de 50000 habitants gèrent leurs services d'eau en régie (Covo-Dahan, 1980). En moins de 10 ans, la DSP gagne 8 millions d'habitants (Paris, Lyon, Toulouse, Toulon, Avignon, Saint-Etienne, Grenoble, Arles, Sète, etc.). Deux facteurs propres à cette époque précipitent ce changement. D'une part, la fin du blocage administratif des prix impose aux régies l'équilibre de leur budget et donc d'importantes augmentations de tarifs auxquelles les communes se soustraient en passant en DSP (Pezon, 2002). D'autre part, la décentralisation offre aux communes de nouvelles libertés contractuelles, dans un contexte marqué par la crise des finances locales, une idéologie favorable à la gestion privée et un nombre restreint d'opérateurs puissants et diversifiés sur l'ensemble des services urbains. La DSP devient monnayable : rien n'interdit aux communes de 'mettre aux enchères' la gestion privée de leurs services d'eau. ${ }^{15}$

En 1993, la loi relative à la prévention de la corruption, dite loi Sapin, cherche à stopper les dérives avérées lors des DSP des services d'eau de Grenoble et Saint-Denis de La Réunion. Elle formalise un processus d'attribution des contrats de DSP et limite -avant que la loi Mazeaud de 1995 ne l'interdise- la perception de droits d'entrée par les communes. Depuis, l'équilibre entre services délégués et régies d'eau s'est stabilisé avec $75 \%$ de la population desservie par des services d'eau potable délégués.

\subsection{L'avènement de nouveaux acteurs menace-il l'hégémonie de l'affermage ?}

Le modèle dominant de la gestion en affermage a permis de contourner l'étroitesse historique et structurelle des services, liée au territoire et/ou à leur spécialisation, par une mutualisation des moyens et compétences nécessaires à leur exploitation. Si la réorganisation des services d'eau urbains laisse apparaître peu d'évolution sur les modes de gestion, elle induit des ruptures originales dans la trajectoire historique que nous avons tracée et les changements qui en découlent peuvent s'envisager à l'aune des liens entre organisation institutionnelle et territoriale et gestion des services. La première cause est l'intégration plus forte de l'eau potable et de l'assainissement dans les communautés ${ }^{16}$. Face à des opérateurs présents dans les deux secteurs, elle leur confère l'envergure suffisante pour développer une maîtrise d'ouvrage plus spécialisée, accroître le contrôle sur les fermiers, voire changer de mode de gestion. La seconde procède de l'héritage sur un territoire communautaire d'opérateurs différents (en vertu de la continuité des contrats), voire de modes de gestion différents, comme dans l'exemple de la communauté urbaine de Nantes. Une régulation des divers opérateurs par comparaison simultanée devient alors envisageable, tout comme l'extension progressive du territoire des régies de centreville, rescapées de la vague des délégations de la fin des années 1980, à l'issue des contrats de délégation.

\footnotetext{
${ }^{13}$ En 1968, la taille moyenne des 1048 communes qui délèguent est de 250 habitants. En 1969, elle augmente brutalement à 1800 , et se stabilise à 1500 habitants jusqu'au début des années 1980 .

${ }^{14}$ Décret du 7 octobre 1968 ; cette possibilité ne sera offerte aux régies qu'en 1975 (décret du 9 juillet 1975 et art.14 de la loi de finances de 1975)

${ }^{15}$ Toulouse détient le record avec la perception d'un droit d'entrée de 437,5 millions de francs pour la DSP de ses services d'eau potable et d'assainissement en 1989

${ }^{16}$ En 2007, 142 communautés de communes (sur 207) et 76 communautés d'agglomérations (sur 85) sont, à l'instar des 14 communautés urbaines, doublement compétentes.
}

Espaces et Sociétés - Usages et Régulations des Eaux Urbaines 139 n4/2009 pp.21-38 
Conjugués à une taille de service plus importante et à la volonté de positionner les nouvelles communautés comme une institution de premier plan, ces deux facteurs contribuent à rééquilibrer la relation entre les collectivités et leurs délégataires et pourraient constituer les ferments d'une inversion de tendance en défaveur de l'affermage.

Les services ruraux ne sont pas exclus de l'évolution, malgré un moindre impact de la loi Chevènement. A la faveur des vagues de décentralisation et du retrait progressif de l'Etat, les Conseils Généraux se positionnent comme financeurs de l'eau potable en milieu rural et conseillers auprès des collectivités (Grandgirard, 2007). Ce rôle leur permet d'orienter la réorganisation des services mais selon des logiques différentes de l'époque de la généralisation de la desserte. A la mobilisation de la ressource en eau, se sont substitués sa préservation, la sécurisation de l'approvisionnement ou le renouvellement des infrastructures qui requièrent une échelle territoriale plus large. Si cette évolution se confirmait, elle contribuerait également à l'émergence de services plus à même de contrôler strictement les délégataires, voire de choisir plus librement leurs modes de gestion.

Outre l'accroissement des échelles d'organisation, ces deux mouvements concomitants concourent à une bipolarisation des services d'eau potable autour des centres urbains denses d'une part et des Départements sur les zones rurales, d'autre part. Les effets économiques de cette évolution sont relativement atténués par les transferts de solidarité des Conseils Généraux ou des Agences de l'Eau. Cependant, comme ce fut le cas pour la création des services et la définition de leurs modes de gestion, l'évolution vers la bipolarisation ne sera pas indépendante des contraintes intangibles pesant sur la ressource en eau et des institutions locales déjà en place.

\section{Conclusion}

Nous avons vu que la singularité des services d'eau potable français tient, certes, à la prépondérance d'une gestion privée mais tout autant à la fragmentation des collectivités chargées d'organiser la distribution d'eau et au financement public voire fiscal des investissements. La desserte généralisée du territoire en eau potable est le fruit d'une action volontariste de l'Etat planificateur qui, après 1945, pour procéder à un aménagement rationalisé de son territoire, a trouvé chez les quelques opérateurs rescapés du socialisme municipal et de la période noire de l'Occupation, des auxiliaires dont le principal atout était de pouvoir exploiter des services en s'affranchissant des limites restrictives imposées par la carte communale et par l'intercommunalité à compétence unique. Les modes de gestion sont intimement liés aux règles et aux échelles d'organisation, qui méritent donc une attention plus particulière. Les mutations des services urbains pourraient à l'avenir conduire à une redéfinition des modes de gestion prépondérants, même si pour l'heure les quelques retours en régie restent l'exception, et que contrairement aux régies directes du début du XXe siècle, les nouvelles régies urbaines doivent équilibrer leurs recettes et leurs dépenses sans subvention du budget général ${ }^{17}$.

Il est intéressant de noter que la distribution d'eau potable à domicile s'est développée autour de deux espaces territoriaux antithétiques : le local et le national. L'élection directe des élus municipaux selon le principe 'un homme, une voix', en 1884, a sans conteste focalisé l'attention des décideurs sur une forme de service répondant aux besoins du plus grand nombre. L'organisation et la gestion des services d'eau n'est pourtant pas un pur arrangement local ; loin d'être absent, le niveau national a façonné les règles de coopération intercommunale, d'administration du service et de solidarité, et il a pesé sur les choix de gestion des collectivités locales.

Si le 'débat public-privé' parait simplificateur, opposer frontalement l'Etat et le local est tout autant réducteur. Les syndicats de communes ont d'abord constitué un moyen de mutualiser la ressource, se limitant parfois à la compétence 'production'; leur échelle s'est adaptée à l'envergure des problèmes à résoudre sans aller toutefois dépasser les frontières départementales ${ }^{18}$. Celles-ci ont conjointement servi de cadre aux ingénieurs des services territoriaux de l'Etat pour planifier la desserte généralisée du territoire. Le relais pris ensuite par les Conseils Généraux ne dément pas l'importance de cette échelle. Enfin les opérateurs ont défini leurs territoires

\footnotetext{
${ }^{17}$ Ce sera le cas pour l'établissement public à caractère industriel et commercial 'Eau de Paris' (régie personnalisée) issu de la remunicipalisation du service d'eau.

${ }^{18}$ A l'exception de situations où des compagnies d'aménagement ont été crées, telles que le Canal de Provence qui apporte l'eau de la Durance et du Verdon à Marseille et dans le Var.
} 
d'intervention en dehors des limites administratives, empilant plusieurs niveaux de mutualisations entre services morcelés. Les divers défis économiques, environnementaux et sociaux auxquels ils seront confrontés à l'avenir conduiront-ils à maintenir de multiples niveaux et territoires d'organisations ad hoc, voire à les multiplier ? 


\section{Références}

BIPE/FP2E, 2008, Les services collectifs d'eau et d'assainissement en France. Données économiques, sociales et techniques, Janvier, 3 ème édition, 52p.

BouRJOL M., 1993, (sous la direction de), Intercommunalité et coopération intercommunale, Paris, Librairie générale de droit et de jurisprudence, 220p.

BuREL J., 1912, La régie directe considérée du point de vue de l'hygiène dans les villes, la question à Lyon, Thèse pour le doctorat en droit, Paris, Ed. A. Rousseau.

CANNEVA, G., 2007, Vers un système d'information des services d'eau et d'assainissement, rapport pour le Medd, Laboratoire Gestion de l'Eau et de l'Assainissement, juin, 37 p.

Covo-Dahan P., 1980, Sous-traitance d'un service public communal. Stratégie d'implantation sur le marché de l'eau, Thèse en économie, Université Paris-Dauphine.

Delamarre, F, Auriac, F. Durand-Dastes, P. Brossier, 1992, Les services de réseaux en France. Intercommunalité et mode de gestion, GIP Reclus.

ERNST \& YOUNG, 2004, Etude relative au calcul de la récupération des coûts des services liés à l'utilisation de l'eau pour les districts hydrographiques français, Rapport pour le Medd.

Goubert, J.-P. (1987). La conquête de l'eau. L'avènement de la santé à l'âge industriel Paris, Hachette Pluriel.

GUILLERME A. (1984). « Capter, clarifier, transporter l'eau en France (1800-1850) », Les annales de la recherche urbaine $\mathrm{n}^{\circ} 23-24$

GRANDGIRARD, A. (2007). De la gestion intégrée comme doctrine à l'intégration comme défi de gestion, Doctorat en Sciences de Gestion, Ecole des Mines de Paris. 285 p.

IFEN, 2007, Les services publics de l'eau en 2004 - Volet eau potable. Collection Les dossiers, n $7,34 p$.

LEYDET V., 1936, Le syndicat de communes, Paris, Librairie technique et économique.

LoOSDREGT H.B., 1990, «Services publics locaux, l'exemple de l'eau », Actualité juridique-Droit administratif vol. 11, 20 novembre.

LORIFERNE H., 1987, (sous la direction de), 40 ans de politique de l'eau en France, Paris, Economica.

Ministère de l'Agriculture, Direction générale du Génie rural et de l'Hydraulique agricole, 1959, Trois enquêtes sur les services publics ruraux en France, Tome 1. Inventaire des distributions rurales d'eau potable en France au ler janvier 1954, Paris, Imprimerie Nationale.

MONSARRAT G, 1920, Contrats et concessions des communes et des établissements communaux de bienfaisance, Paris, Bibliothèque municipale et rurale.

Murard L., Zylberman P., 1996, L’hygiène dans la République. La santé publique en France ou l'utopie contrariée 1870-1918, Paris, Fayard.

PERRIN B., 1994, La coopération intercommunale : bilan et perspectives, Paris, Berger Levrault.

PEZON C., 2000, Le service d'eau potable en France de 1850 à 1995, Paris, Cnam.

PEZON, C., 2002, «La dérégulation discrète de la distribution d'eau potable en France et l'émergence d'un nouvel acteur collectif, les abonnés », Flux n 48-49, 15 p.

PezOn C., Petitet S., 2004, «Histoire de l'intercommunalité en France (1890-1999): la distribution d'eau potable en question », communication au Colloque "Les territoires de l'eau", Réseau Développement Durable et Territoires Fragiles, Université d'Artois, 26 mars.

PEZON, C., 2007, “The role of users' cases in drinking water services development and regulation in France: a historical perspective", Utilities Policy, vol. 15, n² 2, pp. $110-120$.

VIGUIER, J., 1992, Les régies des collectivités locales, Paris, Economica. 